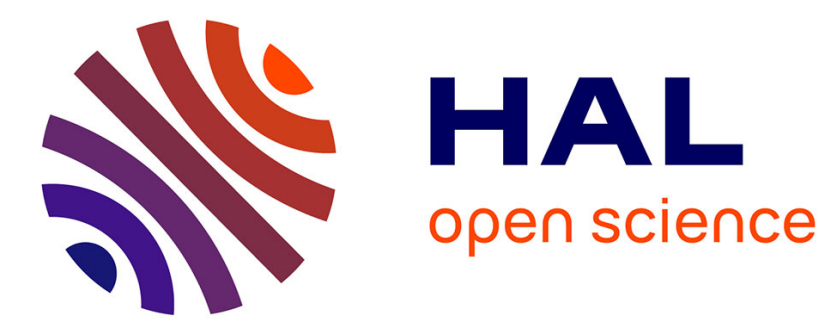

\title{
LOGARITHMIC LITTLEWOOD-PALEY \\ DECOMPOSITION AND APPLICATIONS TO \\ ORLICZ SPACES
}

Hajer Bahouri

\section{- To cite this version:}

Hajer Bahouri. LOGARITHMIC LITTLEWOOD-PALEY DECOMPOSITION AND APPLICATIONS TO ORLICZ SPACES. 2015. hal-01113292

\section{HAL Id: hal-01113292 \\ https://hal.science/hal-01113292}

Preprint submitted on 4 Feb 2015

HAL is a multi-disciplinary open access archive for the deposit and dissemination of scientific research documents, whether they are published or not. The documents may come from teaching and research institutions in France or abroad, or from public or private research centers.
L'archive ouverte pluridisciplinaire HAL, est destinée au dépôt et à la diffusion de documents scientifiques de niveau recherche, publiés ou non, émanant des établissements d'enseignement et de recherche français ou étrangers, des laboratoires publics ou privés. 


\title{
LOGARITHMIC LITTLEWOOD-PALEY DECOMPOSITION AND APPLICATIONS TO ORLICZ SPACES
}

\author{
HAJER BAHOURI
}

\begin{abstract}
This paper is devoted to the construction of a logarithmic Littlewood-Paley decomposition. The approach we adopted to carry out this construction is based on the notion introduced in [9] of being log-oscillating with respect to a scale. The relevance of this theory is illustrated on several examples related to Orlicz spaces.
\end{abstract}

\section{Contents}

\begin{tabular}{|lr|}
\hline 1. Introduction and statement of the results & 1 \\
\hline 1.1. Setting of the problem & 1 \\
\hline $1.2 . \quad$ Background materia] & 3 \\
\hline 1.3. Main results & 6 \\
\hline 1.4. Lavout of the paper & 9 \\
\hline 2. Proof of Bernstein inequalities & 9 \\
\hline 2.1. Proof of Lemma 1.12 & 10 \\
2.2. Proof of Lemma 1.13 & 11 \\
\hline 3. Logarithmic Sobolev embeddings & 12 \\
\hline 3.1. Sobolev embedding of logarithmic Besov spaces into the Orlicz spaces & 12 \\
\hline 3.2. A refined radial estimate & 14 \\
\hline References & 15
\end{tabular}

\section{INTRODUCTION AND STATEMENT OF THE RESUlts}

1.1. Setting of the problem. The aim of this paper is to construct a logarithmic Littlewood-Paley decomposition taking advantage of the notion introduced in [9] of being log-oscillating with respect to a scale. Our main motivation to carry out this decomposition is that it provides a new point of view in the understanding of the Orlicz spaces $\mathcal{L}\left(\mathbb{R}^{2 N}\right)$. Recall that generally the Orlicz spaces are defined as follows (for a complete presentation and more details, we refer the reader to [24]):

Definition 1.1. Let $\phi: \mathbb{R}^{+} \rightarrow \mathbb{R}^{+}$be a convex increasing function such that

$$
\phi(0)=0=\lim _{s \rightarrow 0^{+}} \phi(s) \quad \text { and } \quad \lim _{s \rightarrow \infty} \phi(s)=\infty .
$$

We say that a measurable function $u: \mathbb{R}^{d} \rightarrow \mathbb{C}$ belongs to $L^{\phi}$ if there exists $\lambda>0$ such that

$$
\int_{\mathbb{R}^{d}} \phi\left(\frac{|u(x)|}{\lambda}\right) d x<\infty
$$

We denote then

$$
\|u\|_{L^{\phi}}=\inf \left\{\lambda>0, \quad \int_{\mathbb{R}^{d}} \phi\left(\frac{|u(x)|}{\lambda}\right) d x \leq 1\right\} .
$$

The space $\mathcal{L}\left(\mathbb{R}^{2 N}\right)$ it will be question in this paper is the Orlicz space associated to the function $\phi(s)=\mathrm{e}^{s^{2}}-1$. This space intervenes via the following sharp Moser-Trudinger type inequalities (see [1, 2, 25, 27, for further details):

Date: January 30, 2015.

Key words and phrases. Orlicz; log-oscillating; Littlewood-Paley decomposition. 


\section{Proposition 1.2.}

$$
\sup _{\|u\|_{H^{N}\left(\mathbb{R}^{2 N}\right)} \leq 1} \int_{\mathbb{R}^{2 N}}\left(\mathrm{e}^{\beta_{N}|u(x)|^{2}}-1\right) d x<\infty
$$

where $\beta_{N}=\frac{2 N \pi^{2 N} 2^{2 N}}{\omega_{2 N-1}}$, with $\omega_{2 N-1}=\frac{2 \pi^{N}}{(N-1) !}$ the measure of the unit sphere $\mathbb{S}^{2 N-1}$.

Indeed, Estimate (1.2) leads obviously to the Sobolev embedding

$$
H^{N}\left(\mathbb{R}^{2 N}\right) \hookrightarrow \mathcal{L}\left(\mathbb{R}^{2 N}\right),
$$

whose lack of compactness has been investigated by several authors (for further details, we refer to [7, 8, 12, 21, 22, 26]). Since the works of P. -L. Lions (21, 22]), it is well understood that the defect of compactness of the Sobolev embedding (1.3) in 2D is due to two reasons. The first reason is the lack of compactness at infinity that can be illustrated by the sequence $u_{n}(x)=\varphi\left(x+x_{n}\right)$, where $0 \neq \varphi \in \mathcal{D}$ and $\left|x_{n}\right| \rightarrow \infty$, and the second reason is of concentration-type and can be highlighted by the example by Moser (see [21, 22, 23]) defined by:

$$
f_{\alpha_{n}}(x)=\left\{\begin{array}{clll}
\sqrt{\frac{\alpha_{n}}{2 \pi}} & \text { if } & |x| \leq \mathrm{e}^{-\alpha_{n}}, \\
-\frac{\log |x|}{\sqrt{2 \alpha_{n} \pi}} & \text { if } & \mathrm{e}^{-\alpha_{n}} \leq|x| \leq 1, \\
0 & \text { if } & |x| \geq 1,
\end{array}\right.
$$

where $\underline{\alpha}:=\left(\alpha_{n}\right)$ is a sequence of positive real numbers going to infinity. Recall that by straightforward computations (detailed for instance in [7]):

$$
f_{\alpha_{n}} \rightarrow 0 \quad \text { in } \quad H^{1}\left(\mathbb{R}^{2}\right) \quad \text { and } \quad\left\|f_{\alpha_{n}}\right\|_{\mathcal{L}\left(\mathbb{R}^{2}\right)} \rightarrow \frac{1}{\sqrt{4 \pi}}, \quad \text { as } \quad n \rightarrow \infty
$$

Contrary to the case of the elementary concentrations involved in the framework studied by P. Gérard in [18. (see also [3, 15, 19]) concerning the critical Sobolev embedding

$$
\dot{H}^{s}\left(\mathbb{R}^{d}\right) \hookrightarrow L^{p}\left(\mathbb{R}^{d}\right),
$$

with $0 \leq s<d / 2$ and $p=2 d /(d-2 s)$, the frequencies of the sequence $\left(f_{\alpha_{n}}\right)_{n \geq 0}$ are spread. More precisely with the vocabulary of [18] (see also Definition 1.5] in this paper), the sequence $\left(\nabla f_{\alpha_{n}}\right)_{n \geq 0}$ is "unrelated" to any scale. As it has been emphasized in 18 that the characteristic of being unrelated to any scale is measured using the Besov norm $\dot{B}_{2, \infty}^{0}$ (see for example [6] for a detailed exposition on Besov spaces), this gives rise to

$$
\left\|\nabla f_{\alpha_{n}}\right\|_{\dot{B}_{2, \infty}^{0}\left(\mathbb{R}^{2}\right)} \longrightarrow 0, \quad \text { as } \quad n \rightarrow \infty .
$$

Actually in 10, we have generalized this phenomenon to the 2ND case, which implies that the classical Besov space $B_{2, \infty}^{N}\left(\mathbb{R}^{2 N}\right)$ does not embed into the Orlicz space $\mathcal{L}\left(\mathbb{R}^{2 N}\right)$. We will rather see in Paragraph 3.1 that a more suitable Besov space built up from the logarithmic Littlewood-Paley decomposition embeds in the Orlicz space.

Let us end this paragraph by noting that in general it can be inferred from Moser-Trudinger inequalities (1.2) that

$$
H^{N}\left(\mathbb{R}^{2 N}\right) \hookrightarrow L^{\phi_{p}}\left(\mathbb{R}^{2 N}\right), \quad \forall p \geq 1,
$$

where $L^{\phi_{p}}\left(\mathbb{R}^{2 N}\right)$ denotes the Orlicz space associated to the function

$$
\phi_{p}(s)=\mathrm{e}^{\mathrm{s}^{2}}-\sum_{\mathrm{k}=0}^{\mathrm{p}-1} \frac{\mathrm{s}^{2 \mathrm{k}}}{\mathrm{k} !} .
$$


1.2. Background material. The notion of log-oscillating sequences have proved to be very efficient in the characterization of the lack of compactness of the critical Sobolev embedding (1.3) in the 2ND general case (see [10] for further details). Let us then start by recalling this notion and some basic related facts:

Definition 1.3. Let $v:=\left(v_{n}\right)_{n \geq 0}$ be a bounded sequence in $L^{2}\left(\mathbb{R}^{d}\right)$ and $\underline{\alpha}:=\left(\alpha_{n}\right)_{n \geq 0}$ be a sequence of positive real numbers going to infinity.

- The sequence $v$ is said $\underline{\alpha} \log$-oscillating if 1

$$
\limsup _{n \rightarrow \infty}\left(\int_{|\xi| \leq \mathrm{e}^{\frac{\alpha_{n}}{R}}}\left|\widehat{v_{n}}(\xi)\right|^{2} d \xi+\int_{|\xi| \geq \mathrm{e}^{R \alpha_{n}}}\left|\widehat{v_{n}}(\xi)\right|^{2} d \xi\right) \stackrel{R \rightarrow \infty}{\longrightarrow} 0 .
$$

- The sequence $v$ is said log-unrelated to the scale $\underline{\alpha}$ if for any real numbers $b>a>0$

$$
\int_{\mathrm{e}^{a \alpha_{n}} \leq|\xi| \leq \mathrm{e}^{b \alpha_{n}}}\left|\widehat{v_{n}}(\xi)\right|^{2} d \xi \stackrel{n \rightarrow \infty}{\longrightarrow} 0 .
$$

\section{Remarks 1.4.}

- Clearly the notion of log-oscillating is only relevant for scales $\left(\alpha_{n}\right)_{n \geq 0}$ converging towards infinity.

- Inspired by the counter-example of P. Gérard in [18, one can prove the existence of sequences log-unrelated to any scale which nevertheless do not converge strongly to 0 in $L^{2}\left(\mathbb{R}^{d}\right)$. To be convinced, let us consider in $L^{2}\left(\mathbb{R}^{d}\right)$ the sequence $\left(v_{n}\right)_{n \geq 3}$ defined by:

$$
\widehat{v_{n}}(\xi):=\frac{1}{\sqrt{\log (\log n)}} \frac{\widehat{\psi}\left(\frac{\xi}{n}\right)}{\sqrt{\left(1+||^{d}|\log | \xi||\right)}},
$$

where $\psi$ is a function in $\mathcal{S}\left(\mathbb{R}^{d}\right)$ satisfying $\int_{\mathbb{R}^{d}} \psi(x) d x \neq 0$.

On the one hand by straightforward computations, we get for any sequence $\left(\alpha_{n}\right)$ tending to infinity and any real numbers $b>a>0$

$$
\int_{\mathrm{e}^{a \alpha_{n}} \leq|\xi| \leq \mathrm{e}^{b \alpha_{n}}}\left|\widehat{v_{n}}(\xi)\right|^{2} d \xi \leq \frac{C_{\psi}}{\log (\log n)} \int_{\mathrm{e}^{a \alpha_{n}}}^{\mathrm{e}^{b \alpha_{n}}} \frac{d \rho}{\rho \log (\rho)} .
$$

Performing the change of variables $\rho=\mathrm{e}^{u}$, we easily deduce that

$$
\int_{\mathrm{e}^{a \alpha_{n}} \leq|\xi| \leq \mathrm{e}^{b \alpha_{n}}}\left|\widehat{v_{n}}(\xi)\right|^{2} d \xi \leq \frac{C_{\psi} \log \left(\frac{b}{a}\right)}{\log (\log n)} \stackrel{n \rightarrow \infty}{\longrightarrow} 0,
$$

which ensures that the sequence $\left(v_{n}\right)$ is log-unrelated to any scale tending to infinity.

In other respects for any fixed $M$, we have

$$
\int_{|\xi| \leq M}\left|\widehat{v_{n}}(\xi)\right|^{2} d \xi \lesssim \frac{\|\widehat{\psi}\|_{L^{\infty}\left(\mathbb{R}^{d}\right)}^{2} M^{d}}{\log (\log n)} \stackrel{n \rightarrow \infty}{\longrightarrow} 0,
$$

which implies that the sequence $\left(v_{n}\right)$ is log-unrelated to any bounded scale.

On the other hand by Fourier-Plancherel formula, we have

with

$$
\left\|v_{n}\right\|_{L^{2}\left(\mathbb{R}^{d}\right)}^{2}=\frac{(2 \pi)^{-d}}{\log (\log n)} \int_{\mathbb{R}^{d}} \frac{\left|\widehat{\psi}\left(\frac{\xi}{n}\right)\right|^{2} d \xi}{\left(1+|\xi|^{d}|\log | \xi||\right)}=\mathcal{I}_{n}^{1}+\mathcal{I}_{n}^{2},
$$

$$
\mathcal{I}_{n}^{1}:=\frac{(2 \pi)^{-d}}{\log (\log n)} \int_{|\xi| \leq n} \frac{\left|\widehat{\psi}\left(\frac{\xi}{n}\right)\right|^{2} d \xi}{\left(1+|\xi|^{d}|\log | \xi||\right)}
$$

\footnotetext{
$1_{\text {where }} \widehat{u}$ denotes the Fourier transform of $u$ defined by: $\widehat{u}(\xi)=\int_{\mathbb{R}^{2 N}} \mathrm{e}^{-i x \cdot \xi} u(x) d x$.
} 
Performing the change of variables $\xi=n \eta$, we get by applying Lebesgue theorem

$$
\mathcal{I}_{n}^{2}=\frac{(2 \pi)^{-d}}{\log (\log n)} \int_{|\eta| \geq 1} \frac{|\widehat{\psi}(\eta)|^{2} d \eta}{\left(\frac{1}{n^{d}}+|\eta|^{d}|\log | n \eta||\right)} \stackrel{n \rightarrow \infty}{\longrightarrow} 0
$$

Since $\widehat{\psi}(0)=\int_{\mathbb{R}^{d}} \psi(x) d x \neq 0$, we obtain making use again of the change of variables $\xi=n \eta$

$$
\mathcal{I}_{n}^{1}=\frac{(2 \pi)^{-d}}{\log (\log n)} \int_{|\eta| \leq 1} \frac{|\widehat{\psi}(\eta)|^{2} d \eta}{\left(\frac{1}{n^{d}}+|\eta|^{d}|\log | n \eta||\right)}=\frac{(2 \pi)^{-d}}{\log (\log n)} \int_{|\eta| \leq 1} \frac{\left(|\widehat{\psi}(0)|^{2}+\mathcal{O}(|\eta|)\right) d \eta}{\left(\frac{1}{n^{d}}+|\eta|^{d}|\log | n \eta||\right)},
$$

which, by straightforward computations, implies that

$$
\mathcal{I}_{n}^{1}=\frac{(2 \pi)^{-d}|\widehat{\psi}(0)|^{2}}{\log (\log n)} \int_{\frac{\mathrm{e}}{n} \leq|\eta| \leq 1} \frac{d \eta}{\left(\frac{1}{n^{d}}+|\eta|^{d}|\log | n \eta||\right)}+\circ(1) \cdot
$$

We deduce that

$$
\mathcal{I}_{n}^{1} \stackrel{n \rightarrow \infty}{\longrightarrow}(2 \pi)^{-d} \omega_{d-1}\left|\int_{\mathbb{R}^{d}} \psi(x) d x\right|^{2},
$$

where $\omega_{d-1}$ denotes the measure of the unit sphere $\mathbb{S}^{d-1}$.

Invoking (1.11) and (1.12), we infer that

$$
\left\|v_{n}\right\|_{L^{2}\left(\mathbb{R}^{d}\right)}^{2} \stackrel{n \rightarrow \infty}{\longrightarrow}(2 \pi)^{-d} \omega_{d-1}\left|\int_{\mathbb{R}^{d}} \psi(x) d x\right|^{2},
$$

which ends the proof of the claim.

These notions of being log-oscillating with respect to a scale and of being log-unrelated to any scale are a natural adaptation to Orlicz spaces setting of the vocabulary of P. Gérard introduced in [18] as follows:

Definition 1.5. Let $v:=\left(v_{n}\right)_{n \geq 0}$ be a bounded sequence in $L^{2}\left(\mathbb{R}^{d}\right)$ and $\underline{h}:=\left(h_{n}\right)_{n \geq 0}$ be a sequence of positive real numbers.

- The sequence $v$ is said $\underline{h}$-oscillating if

$$
\limsup _{n \rightarrow \infty}\left(\int_{h_{n}|\xi| \leq \frac{1}{R}}\left|\widehat{v_{n}}(\xi)\right|^{2} d \xi+\int_{h_{n}|\xi| \geq R}\left|\widehat{v_{n}}(\xi)\right|^{2} d \xi\right) \stackrel{R \rightarrow \infty}{\longrightarrow} 0 .
$$

- The sequence $v$ is said unrelated to the scale $\underline{h}$ if for any reals $b>a>0$

$$
\int_{a \leq h_{n}|\xi| \leq b}\left|\widehat{v_{n}}(\xi)\right|^{2} d \xi \stackrel{n \rightarrow \infty}{\longrightarrow} 0 .
$$

Since our first aim in this paper is to construct a logarithmic Littlewood-Paley decomposition, let us recall the definition of the classical dyadic partition of unity on $\mathbb{R}^{d}$ (we refer for instance to [6, 13, 14, 28, and the references therein for more details).

Definition 1.6. Let $\mathcal{C}$ be the annulus $\left\{\xi \in \mathbb{R}^{d} / 3 / 4 \leq|\xi| \leq 8 / 3\right\}$. There exist two radial functions $\chi$ and $\varphi$ valued in the interval $[0,1]$, belonging respectively to $\mathcal{D}(B(0,4 / 3))$ and to $\mathcal{D}(\mathcal{C})$, and such that

$$
\begin{gathered}
\forall \xi \in \mathbb{R}^{d}, \chi(\xi)+\sum_{j \geq 0} \varphi\left(2^{-j} \xi\right)=1 \text { and } \\
\forall \xi \in \mathbb{R}^{d} \backslash\{0\}, \sum_{j \in \mathbf{Z}} \varphi\left(2^{-j} \xi\right)=1 .
\end{gathered}
$$

\section{Remarks 1.7.}


- For all $u$ in $\mathcal{S}^{\prime}\left(\mathbb{R}^{d}\right)$, we have 2

$$
u=\sum_{j} \Delta_{j} u,
$$

where the nonhomogeneous dyadic blocks $\Delta_{j}$ are defined by

$$
\Delta_{j} u=0 \text { if } j \leq-2, \Delta_{-1} u=\chi(D) u \text { and } \Delta_{j} u=\varphi\left(2^{-j} D\right) u \text { if } j \geq 0 .
$$

Note that Identity (1.17) also assumes the form

$$
u=\lim _{j \rightarrow \infty} \sum_{j^{\prime} \leq j-1} \Delta_{j^{\prime}} u=\lim _{j \rightarrow \infty} S_{j} u \quad \text { in } \quad \mathcal{S}^{\prime}\left(\mathbb{R}^{d}\right),
$$

where the nonhomogeneous low frequency cut-off operator $S_{j}$ writes

$$
S_{j} u=\sum_{-1 \leq j^{\prime} \leq j-1} \Delta_{j^{\prime}} u=\chi\left(2^{-j} D\right) u \quad \text { for } \quad j \geq 0 .
$$

- Equality (1.17) is not valid for all u in $\mathcal{S}^{\prime}\left(\mathbb{R}^{d}\right)$ for homogeneous dyadic blocks $\dot{\Delta}_{j}$ defined by

$$
\dot{\Delta}_{j} u=\varphi\left(2^{-j} D\right) u \quad \text { for } \quad j \in \mathbf{Z} .
$$

It clearly fails for nonzero polynomials. However, it holds true in $\mathcal{S}_{\mathrm{h}}^{\prime}\left(\mathbb{R}^{d}\right)$ the subspace of tempered distributions u satisfying $\left\|\dot{S}_{j} u\right\|_{L^{\infty}\left(\mathbb{R}^{d}\right)} \stackrel{j \rightarrow-\infty}{\longrightarrow} 0$, where $\dot{S}_{j}$ designates the homogeneous low frequency cut-off operator defined by

$$
\dot{S}_{j} u=\sum_{j^{\prime} \leq j-1} \dot{\Delta}_{j^{\prime}} u \quad \text { for } \quad j \in \mathbf{Z} .
$$

- Decomposition (1.17), which supplies an elementary device for splitting a possibly rough function into a sequence of spectrally localized smooth functions, allows among other to define a wide class of function spaces like Besov spaces and to provide elementary and elegant proofs of various inequalities such as refined Sobolev and Hardy inequalities. We can consult Chapter 2 in [6] for an overview of this theory in the classical case.

- Recall that for $s \in \mathbb{R}$ and $1 \leq p, r \leq \infty$, the nonhomogeneous Besov space $B_{p, r}^{s}\left(\mathbb{R}^{d}\right)$ is the set of all tempered distributions $u$ so that

$$
\|u\|_{B_{p, r}^{s}\left(\mathbb{R}^{d}\right)}:=\left\|\left(2^{j s}\left\|\Delta_{j} u\right\|_{L^{p}}\right)_{j \in \mathbf{Z}}\right\|_{\ell^{r}(\mathbf{Z})}<\infty
$$

and the homogeneous Besov space $\dot{B}_{p, r}^{s}\left(\mathbb{R}^{d}\right)$ is the set of tempered distributions belonging to $\mathcal{S}_{\mathrm{h}}^{\prime}\left(\mathbb{R}^{d}\right)$ such that

$$
\|u\|_{\dot{B}_{p, r}^{s}\left(\mathbb{R}^{d}\right)}:=\left\|\left(2^{j s}\left\|\dot{\Delta}_{j} u\right\|_{L^{p}}\right)_{j \in \mathbf{Z}}\right\|_{\ell^{r}(\mathbf{Z})}<\infty .
$$

Thus Estimate (1.6) also reads

$$
\sup _{j \in \mathbf{Z}}\left\|\dot{\Delta}_{j} \nabla f_{\alpha_{n}}\right\|_{L^{2}\left(\mathbb{R}^{2}\right)} \longrightarrow 0, \quad \text { as } \quad n \rightarrow \infty .
$$

- Property (1.18) highlights the fact that the example by Moser is spread in frequency. Note that it was proved in [9] that the sequence $\left(f_{\alpha_{n}}\right)$ can be written under the form:

$$
f_{\alpha_{n}}(x)=\widetilde{f_{\alpha_{n}}}(x)+\mathrm{r}_{n}(x),
$$

with $\left\|\mathrm{r}_{n}\right\|_{H^{1}\left(\mathbb{R}^{2}\right)} \stackrel{n \rightarrow \infty}{\longrightarrow} 0$ and

$$
\widetilde{f_{\alpha_{n}}}(x)=\frac{1}{(2 \pi)^{2}} \sqrt{\frac{2 \pi}{\alpha_{n}}} \int_{\mathbb{R}^{2}} \mathrm{e}^{i x \cdot \xi} \frac{1}{|\xi|^{2}} \varphi\left(\frac{\log |\xi|}{\alpha_{n}}\right) d \xi,
$$

where $\varphi(\eta)=\mathbf{1}_{[0,1]}(\eta)$.

\footnotetext{
$2_{\text {we recall that }} \mathcal{F}(\Theta(D) u)(\xi)=\Theta(\xi) \mathcal{F}(u)(\xi)$, with $\mathcal{F}$ the Fourier transform.
} 
- Obviously, we have

$$
\mathcal{F}\left(\widetilde{f_{\alpha_{n}}}\right)(\xi)=\sqrt{\frac{2 \pi}{\alpha_{n}}} \frac{\mathbf{1}_{\left[1, \mathrm{e}^{\left.\alpha_{n}\right]}\right.}(|\xi|)}{|\xi|^{2}},
$$

which shows that the spectrum of $f_{\alpha_{n}}$ is spread over the disk of radius $\mathrm{e}^{\alpha_{n}}$ centered at the origin.

- More generally, it has been emphasized in [10] that the lack of compactness of the Sobolev embedding (1.3) is displayed by sequences under the form:

$$
g_{\alpha_{n}}(x)=\frac{C_{N}}{\sqrt{\alpha_{n}}} \int_{|\xi| \geq 1} \frac{\mathrm{e}^{i x \cdot \xi}}{|\xi|^{2 N}} \varphi\left(\frac{\log |\xi|}{\alpha_{n}}\right) d \xi,
$$

with $C_{N}=\frac{1}{(2 \pi)^{N} \sqrt{\omega_{2 N-1}}},\left(\alpha_{n}\right)_{n \geq 0}$ a sequence of positive real numbers going to infinity and $\varphi \not \equiv 0$ in $L^{2}\left(\mathbb{R}_{+}\right)$. It was also proved that $\left(|D|^{N} g_{\alpha_{n}}\right)_{n \geq 0}$ is $\underline{\alpha} \log$-oscillating and that

$$
\lim _{n \rightarrow \infty}\left\|g_{\alpha_{n}}\right\|_{\mathcal{L}\left(\mathbb{R}^{2 N}\right)}=\frac{1}{\sqrt{\beta_{N}}} \max _{s>0} \frac{|\psi(s)|}{\sqrt{s}},
$$

where $\psi(s):=\int_{0}^{s} \varphi(t) d t$.

The basic idea of Littlewood-Paley theory is contained in two fundamental inequalities known as Bernstein inequalities. The first one says that, for a function whose Fourier transform is supported in an annulus of size $\lambda$, differentiate and then take the $L^{p}$ norm amounts to do a dilation of ratio $\lambda$ on the $L^{p}$ norm. The second one specifies that, for such functions, the passage from the $L^{p}$ norm to the $L^{q}$ norm, for $q \geq p \geq 1$, costs $\lambda^{d\left(\frac{1}{p}-\frac{1}{q}\right)}$, which should be understood as a Sobolev embedding. More precisely, we have the following lemma the proof of which can be for instance found in [6]:

Lemma 1.8. Let $\mathcal{C}$ be an annulus and $B$ a ball of $\mathbb{R}^{d}$ centered at the origin. A constant $C$ exists so that, for any nonnegative integer $k$, any couple $(p, q)$ in $[1, \infty]^{2}$ with $q \geq p \geq 1$ and any function $u$ of $L^{p}\left(\mathbb{R}^{d}\right)$, we have

$$
\begin{gathered}
\text { Supp } \widehat{u} \subset \lambda B \Longrightarrow\left\|D^{k} u\right\|_{L^{q}\left(\mathbb{R}^{d}\right)}:=\sup _{|\alpha|=k}\left\|\partial^{\alpha} u\right\|_{L^{q}\left(\mathbb{R}^{d}\right)} \leq C^{k+1} \lambda^{k+d\left(\frac{1}{p}-\frac{1}{q}\right)}\|u\|_{L^{p}\left(\mathbb{R}^{d}\right)} \quad \text { and } \\
\text { Supp } \widehat{u} \subset \lambda \mathcal{C} \Longrightarrow C^{-k-1} \lambda^{k}\|u\|_{L^{p}\left(\mathbb{R}^{d}\right)} \leq\left\|D^{k} u\right\|_{L^{p}\left(\mathbb{R}^{d}\right)} \leq C^{k+1} \lambda^{k}\|u\|_{L^{p}\left(\mathbb{R}^{d}\right)}
\end{gathered}
$$

1.3. Main results. The development of microlocal tools adapted to the framework of problems at hand is an important issue: we refer for instance to the articles 4, 5, 11, 16, 17, where was constructed respectively Littlewood-Paley decompositions on the Heisenberg group, on graded Lie groups and on Lie groups of polynomial growth satisfying properties as Bony's decomposition ([13]) in the euclidean case, which enabled to transpose many classical results to these general settings. One can also mention the work [20] where the construction of an adapted Littlewood-Paley theory to the geometric situation of the Einstein equations allows to reach optimal regularity indexes for the initial data.

Our main goal in this paper is to develop a logarithmic Littlewood-Paley theory taking advantage of the notion introduced in [9] of being log-oscillating with respect to a scale which has already proved to be efficacious in 10 in the framework of Orlicz spaces. For that purpose, let us start by introducing the following definition:

Definition 1.9. Under the notations of Definition 1.6, we define for all $u$ in $\mathcal{S}^{\prime}\left(\mathbb{R}^{d}\right)$ the logarithmic dyadic blocks $\Delta_{j}^{\log }$ by

$$
\Delta_{j}^{\log } u=\varphi\left(2^{-j} \log |D|\right)(1-\widetilde{\chi}(D)) u \quad \text { for } \quad j \in \mathbf{Z},
$$

where $\tilde{\chi}$ is a radial function belonging to $\mathcal{D}(B(0,2))$ and satisfying $\widetilde{\chi}(\xi)=1$ for $|\xi| \leq \frac{3}{2}$, and the low logarithmic frequency cut-off operator $S_{j}^{\log }$ by

$$
S_{j}^{\log } u=\widetilde{\chi}(D) u+\sum_{j^{\prime} \leq j-1} \Delta_{j^{\prime}}^{\log } u=\widetilde{\chi}(D) u+\chi\left(2^{-j} \log |D|\right)(1-\widetilde{\chi}(D)) u \quad \text { for } \quad j \in \mathbf{Z} .
$$


Formally, we have

$$
\operatorname{Id}=\widetilde{\chi}(D)+\sum_{j^{\prime} \in \mathbf{Z}} \Delta_{j^{\prime}}^{\log u} .
$$

Actually as in the usual case, we have the following result which ensures that the logarithmic Littlewood-Paley decomposition (1.21) makes sense in $\mathcal{S}^{\prime}\left(\mathbb{R}^{d}\right)$ :

Proposition 1.10. Let $u$ be in $\mathcal{S}^{\prime}\left(\mathbb{R}^{d}\right)$. Then

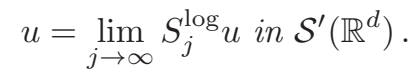

Proof. By classical arguments, one can reduce to the proof of $(1.22)$ in $\mathcal{S}\left(\mathbb{R}^{d}\right)$. Because the Fourier transform is an automorphism of $\mathcal{S}\left(\mathbb{R}^{d}\right)$, we can alternatively prove that for any multi-index $\alpha$ of length $n$, we have

$$
N_{n, \alpha}\left(\left(1-\chi\left(2^{-j} \log |\cdot|\right)\right)(1-\tilde{\chi}) \widehat{u}\right) \stackrel{j \rightarrow \infty}{\longrightarrow} 0,
$$

where $N_{n, \alpha}(f)=\sup _{\mathbb{R}^{d}}(1+|\xi|)^{n}\left|\partial^{\alpha} f(\xi)\right|$.

By virtue of Leibnitz formula, we get (making use of the fact that the function $(1-\tilde{\chi}) \widehat{u}$ is supported on the set $\left.\left\{\xi \in \mathbb{R}^{d} /|\xi| \geq 1\right\}\right)$

$$
\begin{aligned}
N_{n, \alpha}\left(\left(1-\chi\left(2^{-j} \log |\cdot|\right)\right)(1-\widetilde{\chi}) \widehat{u}\right) & \leq \sup _{\mathbb{R}^{d}}(1+|\xi|)^{n}\left\{\left(1-\chi\left(2^{-j} \log |\xi|\right)\right)\left|\partial^{\alpha} \widehat{v}(\xi)\right|\right. \\
& \left.+\sum_{\beta<\alpha} C_{\alpha}^{\beta}\left|\partial^{\alpha-\beta}\left(\chi\left(2^{-j} \log |\xi|\right)\right) \partial^{\beta} \widehat{v}(\xi)\right|\right\},
\end{aligned}
$$

with $\widehat{v}(\xi):=(1-\widetilde{\chi}(\xi)) \widehat{u}(\xi)$.

Since $\widetilde{\chi}(\xi) \equiv 1$ in the ball centered at the origin and of radius $\frac{3}{2}$, we deduce that

$$
N_{n, \alpha}\left(\left(1-\chi\left(2^{-j} \log |\cdot|\right)\right)(1-\tilde{\chi}) \widehat{u}\right) \lesssim 2^{-j} \sup _{|\beta| \leq|\alpha|} N_{n+1, \beta}((1-\tilde{\chi}) \widehat{u}),
$$

which ends the proof of the result.

\section{Remarks 1.11.}

- As emphasized in Remarks 1.4, the notion of log-oscillating with respect to a scale is only relevant for scales tending to infinity. This justifies the fact that the definition of the logarithmic dyadic blocks $\Delta_{j}^{\log }$ does not take into account the low frequencies.

- Clearly there is $j_{0} \in \mathbf{Z}$ such that for any function $u$ in $\mathcal{S}^{\prime}\left(\mathbb{R}^{d}\right)$, we have $\Delta_{j}^{\log } u \equiv 0$ for $j \leq j_{0}$. Furthermore for $j \geq j_{0}$, the function $\Delta_{j}^{\log u}$ is spectrally localized in $\mathrm{e}^{2^{j}} \mathcal{C}$, where $\mathcal{C}$ is the annulus introduced in Definition 1.6. This obviously ensures that the sequence $\left(\Delta_{j}^{\log } u\right)_{j \geq j_{0}}$ is $\left(2^{j}\right)_{j \geq j_{0}} \log$-oscillating.

- Since for applications to the Orlicz space, the logarithmic Littlewood-Paley theory is mostly relevant in 2ND case, we shall limit ourselves in what follows to this case.

- Finally, let us point out that

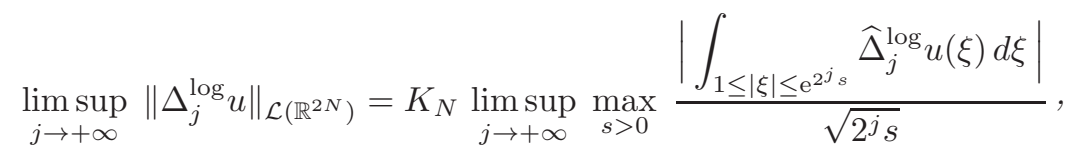

with $K_{N}=\frac{1}{\sqrt{2 N}(2 \pi)^{2 N}} \cdot$ Indeed, by definition 3

$$
\Delta_{j}^{\log } u(x)=\frac{1}{(2 \pi)^{2 N}} \int_{|\xi| \geq 1} \frac{\mathrm{e}^{i x \cdot \xi}}{|\xi|^{2 N}} \widetilde{\Delta}_{j}^{\log } u(\log |\xi|, \omega) d \xi,
$$

\footnotetext{
$3_{\text {where obviously }} \xi=|\xi| \cdot \omega$, with $\omega \in \mathbb{S}^{2 N-1}$.
} 
with $\widetilde{\Delta}_{j}^{\log } u(\log |\xi|, \omega):=|\xi|^{2 N} \widehat{\Delta}_{j}^{\log } u(\xi) \cdot$ Setting

$$
\widetilde{\Delta}_{j}^{\log } u(\log |\xi|, \omega)=\frac{1}{\sqrt{2^{j}}} \Delta_{j}^{\log , b} u\left(\frac{\log |\xi|}{2^{j}}, \omega\right),
$$

we deduce that

$$
\Delta_{j}^{\log } u(x)=\frac{1}{(2 \pi)^{2 N} \sqrt{2^{j}}} \int_{|\xi| \geq 1} \frac{\mathrm{e}^{i x \cdot \xi}}{|\xi|^{2 N}} \Delta_{j}^{\log , b} u\left(\frac{\log |\xi|}{2^{j}}, \omega\right) d \xi .
$$

This ensures in view of Lemma 3.4 in [10] that

$$
\Delta_{j}^{\log } u(x)=\frac{1}{(2 \pi)^{2 N} \sqrt{2^{j}}} \int_{|\xi| \geq 1} \frac{\mathrm{e}^{i x \cdot \xi}}{|\xi|^{2 N}} \Delta_{j}^{\log , \sharp} u\left(\frac{\log |\xi|}{2^{j}}\right) d \xi+\mathrm{r}_{j}(x),
$$

with $\left\|\mathrm{r}_{j}\right\|_{\mathcal{L}\left(\mathbb{R}^{2 N}\right)} \stackrel{j \rightarrow \infty}{\longrightarrow} 0$ and

$$
\Delta_{j}^{\log , \sharp}(t)=\frac{1}{\omega_{2 N-1}} \int_{\mathbb{S}^{2 N-1}} \Delta_{j}^{\log , b} u(t, \omega) d \omega .
$$

In light of (1.20), this gives rise to

$$
\left\|\Delta_{j}^{\log } u\right\|_{\mathcal{L}\left(\mathbb{R}^{2 N}\right)}=\frac{1}{\sqrt{2 N}} \max _{s>0} \frac{\left|\int_{0}^{s} \Delta_{j}^{\log , \sharp} u(t) d t\right|}{\sqrt{s}}+\circ(1),
$$

which by straightforward computations leads to

$$
\left\|\Delta_{j}^{\log } u\right\|_{\mathcal{L}\left(\mathbb{R}^{2 N}\right)}=\frac{1}{\sqrt{2 N}(2 \pi)^{2 N}} \max _{s>0} \frac{\mid \int_{1 \leq|\xi| \leq \mathrm{e}^{j_{s}}} \widehat{\Delta}_{j}^{\log u(\xi) d \xi \mid}}{\sqrt{2^{j} s}}+\circ(1) .
$$

This ends the proof of Claim (1.23).

In order to state the logarithmic Bernstein inequalities in a clear way, let us define the notion of annulus of exponential size. Given $\mathcal{C}=\left\{\xi \in \mathbb{R}^{2 N} / r \leq|\xi| \leq R\right\}$ an annulus of $\mathbb{R}^{2 N}$, we shall denote by $\mathrm{e}^{\mathcal{C}}$ the annulus defined as follows:

$$
\mathrm{e}^{\mathcal{C}}:=\left\{\eta \in \mathbb{R}^{2 N} / \mathrm{e}^{r} \leq|\eta| \leq \mathrm{e}^{R}\right\} .
$$

As in the euclidean case, the first interest of this logarithmic localization procedure in frequency space is that the "logarithmic derivatives" act almost as homothety on distributions the Fourier transform of which is supported in an annulus of exponential size. More precisely, we have the following inequalities which are the counterpart of the second Bernstein inequality stated in Lemma 1.8.

Lemma 1.12. Let $\mathcal{C}$ be an annulus of $\mathbb{R}^{2 N}$ included in the set $\left\{\xi \in \mathbb{R}^{2 N} /|\xi|>1\right\}$. For any nonnegative integer $k$, there exist positive constants $C_{k}$ and $\widetilde{C}_{k}$ so that, for any real number $p \geq 1$ and any function $u$ belonging to $L^{p}\left(\mathbb{R}^{2 N}\right)$ satisfying Supp $\widehat{u} \subset \mathrm{e}^{\lambda \mathcal{C}}$, with $\lambda \geq 1$, we have

$$
\widetilde{C}_{k} \lambda^{k}\|u\|_{L^{p}\left(\mathbb{R}^{2 N}\right)} \leq\left\|(\log |D|)^{k} u\right\|_{L^{p}\left(\mathbb{R}^{2 N}\right)} \leq C_{k} \lambda^{k}\|u\|_{L^{p}\left(\mathbb{R}^{2 N}\right)} .
$$

The generalization of the first part of classical Bernstein inequalities stated in Lemma 1.8 is more challenging. The result we obtain in the logarithmic frame reads differently from that of the classical case, but expresses the same phenomenon, namely that we lose $2 N\left(\frac{1}{p}-\frac{1}{q}\right)$ derivatives in the passage from the $L^{p}$ norm to the $L^{q}$ norm, for $q \geq p \geq 1$. More precisely, our result formulates as follows:

Lemma 1.13. Let $\mathcal{C}$ be an annulus of $\mathbb{R}^{2 N}$ included in the set $\left\{\xi \in \mathbb{R}^{2 N} /|\xi|>1\right\}$. There is a positive real number $b$ so that the following holds. For any nonnegative integer $k$, there exists a positive constant $C_{k}$ such that, for any real number $\lambda \geq 1$, any couple $(p, q)$ in $[1, \infty]^{2}$ with $q \geq p \geq 1$ and any function $u$ belonging to $L^{p}\left(\mathbb{R}^{2 N}\right)$ whose spectrum is included in $\mathrm{e}^{\lambda \mathcal{C}}$, we have

$$
\left\|(\log |D|)^{k} u\right\|_{L^{q}\left(\mathbb{R}^{2 N}\right)} \leq C_{k} \lambda^{k} \mathrm{e}^{2 N \lambda b\left(\frac{1}{p}-\frac{1}{q}\right)}\|u\|_{L^{p}\left(\mathbb{R}^{2 N}\right)} .
$$


Remark 1.14. Contrary to the classical case, the cost of the passage from the $L^{p}$ norm to the $L^{q}$ norm is exponential. This is justified by the fact that the spectrum of the functions considered is of exponential size.

As mentioned above, among the objectives of the Littlewood-Paley theory is to construct functional spaces like Besov spaces and to study their properties. As in the euclidean case, let us define the logarithmic Besov spaces.

Definition 1.15. Let $s$ be a real number, and $(p, r)$ be in $[1, \infty]^{2}$. The logarithmic Besov space $B_{p, r}^{s, \log }\left(\mathbb{R}^{2 N}\right)$ is the subset of tempered distributions $u$ of $\mathcal{S}^{\prime}\left(\mathbb{R}^{2 N}\right)$ such that

$$
\|u\|_{B_{p, r}^{s, \log }\left(\mathbb{R}^{2 N}\right)}:=\|\widetilde{\chi}(D) u\|_{L^{p}\left(\mathbb{R}^{2 N}\right)}+\left\|\left(2^{j s}\left\|\Delta_{j}^{\log } u\right\|_{L^{p}\left(\mathbb{R}^{2 N}\right)}\right)_{j \in \mathbf{Z}}\right\|_{\ell^{r}(\mathbf{Z})}<\infty .
$$

\section{Remarks 1.16.}

- Clearly $L^{2}\left(\mathbb{R}^{2 N}\right)$ coincides with $B_{2,2}^{0, \log }\left(\mathbb{R}^{2 N}\right)$ which implies that

$$
L^{2}\left(\mathbb{R}^{2 N}\right) \hookrightarrow B_{2, \infty}^{0, \log }\left(\mathbb{R}^{2 N}\right) .
$$

- Note also that in view of logarithmic Bernstein inequalities, $(\log |D|)^{k}$ operate on logarithmic Besov spaces $B_{p, r}^{s, \log }\left(\mathbb{R}^{2 N}\right)$, namely that for any real number $s$ and any $(p, q)$ in $[1, \infty]^{2}$, the map

$$
(\log |D|)^{k}: B_{p, r}^{s, \log }\left(\mathbb{R}^{2 N}\right) \longrightarrow B_{p, r}^{s-k, \log }\left(\mathbb{R}^{2 N}\right)
$$

defines a continuous linear functional.

- As in the classical case, the logarithmic cut-off operators $\Delta_{j}^{\log }$ and $S_{j}^{\log }$ are convolution operators on $\mathbb{R}^{2 N}$ defined for $j \in \mathbf{Z}$ by:

$$
\Delta_{j}^{\log } u=h_{j} \star(1-\tilde{\chi}(D)) u,
$$

and

$$
S_{j}^{\log } u=\widetilde{h}_{j} \star(1-\widetilde{\chi}(D)) u .
$$

Contrary to the classical case, the functions $h_{j}$ and $\widetilde{h}_{j}$ are not dilated of integrable functions. However, they belong to $L^{1}\left(\mathbb{R}^{2 N}\right)$ with norms independent of $j$. This property is more challenging than the classical case. Its proof will be given in Paragraph 2.1. In view of Hölder inequalities, it implies that the operators $\Delta_{j}^{\log }$ and $S_{j}^{\log }$ map $L^{p}\left(\mathbb{R}^{2 N}\right)$ into $L^{p}\left(\mathbb{R}^{2 N}\right)$ with norms independent of $j$ and $p$.

- Let us finally note that as in the classical case the definition of the Besov space $B_{p, r}^{s, l o g}$ is independent of the functions $\tilde{\chi}$ and $\varphi$ used for defining the logarithmic dyadic bloks, and changing these functions yields an equivalent norm.

1.4. Layout of the paper. The paper is organized as follows. In Section 2, we prove Bernstein inequalities in the framework of the logarithmic Littlewood-Paley decomposition which are the subject of Lemmas 1.12 and 1.13. Then in Section 3, we state and establish some logarithmic Sobolev embeddings that occur in Orlicz spaces.

We mention that the letter $C$ will be used to denote an absolute constant which may vary from line to line. We also use $A \lesssim B$ to denote an estimate of the form $A \leq C B$ for some absolute constant $C$.

\section{Proof of Bernstein inequalities}

This section is devoted to the proof of Bernstein inequalities in the framework of the logarithmic Littlewood-Paley decomposition. Adapting these fundamental inequalities provides various functional inequalities such as Sobolev embeddings and their refined versions. We will dedicate Section 3 to some functional inequalities which does not arise immediately from an adaptation of the classical framework. 
2.1. Proof of Lemma 1.12, Assuming that $\mathcal{C}=\left\{\xi \in \mathbb{R}^{2 N} / 1<r \leq|\xi| \leq R<b\right\}$, let $\phi$ be a function in $\mathcal{D}(\mathbb{R})$ such that $\operatorname{supp}(\phi) \subset] 1, b[$ and $\phi \equiv 1$ near $] r, R[$. Since the spectrum of $u$ is included in $\mathrm{e}^{\lambda \mathcal{C}}$ with $\lambda \geq 1$, we have $\widehat{u}(\xi)=\phi\left(\lambda^{-1} \log |\xi|\right) \widehat{u}(\xi)$. Thus

$$
(\log |D|)^{k} u=(\log |D|)^{k} g_{\lambda} \star u,
$$

where $\widehat{g_{\lambda}}(\xi)=\phi\left(\lambda^{-1} \log |\xi|\right)$. Applying Young's inequality, we get

$$
\left\|(\log |D|)^{k} u\right\|_{L^{p}} \leq\left\|(\log |D|)^{k} g_{\lambda}\right\|_{L^{1}}\|u\|_{L^{p}} .
$$

But

$$
\mathcal{F}\left((\log |D|)^{k} g_{\lambda}\right)(\xi)=\lambda^{k}\left(\lambda^{-1} \log |\xi|\right)^{k} \phi\left(\lambda^{-1} \log |\xi|\right) .
$$

We are then reduced to estimate the $L^{1}$-norm of the function

$$
g_{k, \lambda}(x):=(2 \pi)^{-2 N} \int_{\mathbb{R}^{2 N}} \mathrm{e}^{i x \cdot \xi}\left(\lambda^{-1} \log |\xi|\right)^{k} \phi\left(\lambda^{-1} \log |\xi|\right) d \xi .
$$

On the one hand

$$
\left|g_{k, \lambda}(x)\right| \lesssim \int_{\mathbb{R}^{2 N}}\left|\phi_{k}\left(\lambda^{-1} \log |\xi|\right)\right| d \xi
$$

with $\phi_{k}(\rho):=\rho^{k} \phi(\rho)$. According to the fact that $\phi_{k} \in \mathcal{D}(] 1, b[)$, this gives rise to

$$
\left|g_{k, \lambda}(x)\right| \lesssim \mathrm{e}^{2 N \lambda b} \text {. }
$$

We deduce that for any positive real number $\delta$, we have

$$
\int_{|x| \leq \delta}\left|g_{k, \lambda}(x)\right| d x \lesssim\left(\delta \mathrm{e}^{\lambda b}\right)^{2 N}
$$

On the other hand by straightforward integrations by parts, we get for any $x \neq 0$

$$
|x|^{2} g_{k, \lambda}(x)=-(2 \pi)^{-2 N} \int_{\mathbb{R}^{2 N}} \mathrm{e}^{i x \cdot \xi} \Delta_{\xi}\left(\phi_{k}\left(\lambda^{-1} \log |\xi|\right)\right) d \xi,
$$

where $\Delta_{\xi}:=\sum_{j=1}^{2 N} \partial_{\xi_{j}}^{2}$. Observing that

$$
\Delta_{\xi}\left(\phi_{k}\left(\lambda^{-1} \log |\xi|\right)\right)=\lambda^{-2} \phi_{k}^{\prime \prime}\left(\lambda^{-1} \log |\xi|\right) \frac{1}{|\xi|^{2}}+\lambda^{-1} \phi_{k}^{\prime}\left(\lambda^{-1} \log |\xi|\right) \frac{2 N-2}{|\xi|^{2}},
$$

we infer that in the $2 \mathrm{D}$ case

$$
\left.|| x\right|^{2} g_{k, \lambda}(x)\left|\lesssim \lambda^{-2} \int_{1}^{\infty}\right| \phi_{k}^{\prime \prime}\left(\lambda^{-1} \log \rho\right) \mid \frac{d \rho}{\rho} \lesssim \lambda^{-1}\left\|\phi_{k}^{\prime \prime}\right\|_{L^{1}} .
$$

Through a second integration by parts, we easily find that for any $\lambda \geq 1$

$$
\left.|| x\right|^{3} g_{k, \lambda}(x)\left|\lesssim \lambda^{-2} \int_{1}^{\infty}\right| H\left(\lambda^{-1} \log \rho\right) \mid \frac{d \rho}{\rho^{2}} \lesssim \lambda^{-2},
$$

where the function $H$ (depending on $k$ ) is a function of $\mathcal{D}(\mathbb{R})$.

Taking advantage of Formula (2.2) and the fact that $\Delta_{\xi}^{(N-1)}\left(|\xi|^{-2}\right)=c_{N} \delta_{0}$ in $\mathbb{R}^{2 N}$ when $N>1$, we get by repeated integrations by parts for any $\lambda \geq 1$

$$
\left.|| x\right|^{2 N} g_{k, \lambda}(x) \mid \lesssim \lambda^{-2} \int_{|\xi| \geq 1} H_{2 N}\left(\lambda^{-1} \log |\xi|\right) \frac{d \xi}{|\xi|^{2 N}} \lesssim \lambda^{-1}
$$

and

$$
\left.|| x\right|^{2 N+1} g_{k, \lambda}(x) \mid \lesssim \lambda^{-2} \int_{|\xi| \geq 1} H_{2 N+1}\left(\lambda^{-1} \log |\xi|\right) \frac{d \xi}{|\xi|^{2 N+1}} \lesssim \lambda^{-2} \lesssim 1
$$

where the functions $H_{2 N}$ and $H_{2 N+1}$ (also depending on $k$ ) are functions belonging to $\mathcal{D}(\mathbb{R})$. This implies that

$$
\int_{|x| \geq 1}\left|g_{k, \lambda}(x)\right| d x \lesssim 1
$$


and for any $0<\delta \leq 1$

$$
\int_{\delta \leq|x| \leq 1}\left|g_{k, \lambda}(x)\right| d x \lesssim \lambda^{-1} \int_{\delta}^{1} \frac{d \rho}{\rho} \lesssim-\lambda^{-1} \log (\delta) .
$$

Choosing $\delta=\mathrm{e}^{-\lambda b}$ and invoking (2.1), (2.5) and (2.6), we infer that

$$
\left\|g_{k, \lambda}\right\|_{L^{1}\left(\mathbb{R}^{2 N}\right)} \lesssim 1
$$

which ends the proof of the right hand side of the assertion.

Once observed that the function $u$ can be recast under the form

$$
u=(\log |D|)^{-k} g_{\lambda} \star(\log |D|)^{k} u,
$$

we end the proof of the result.

2.2. Proof of Lemma 1.13. The proof of this lemma goes the same lines as the proof of Lemma 1.12. Taking advantage of the fact that the spectrum of the function $u$ is included in $\mathrm{e}^{\lambda \mathcal{C}}$ with $\lambda \geq 1$, we find that $\widehat{u}(\xi)=\phi\left(\lambda^{-1} \log |\xi|\right) \widehat{u}(\xi)$, where $\phi$ is a function of $\mathcal{D}(\mathbb{R})$ chosen as above.

Therefore

$$
(\log |D|)^{k} u=(\log |D|)^{k} g_{\lambda} \star u,
$$

where $\widehat{g_{\lambda}}(\xi)=\phi\left(\lambda^{-1} \log |\xi|\right)$. Thanks to Young inequalities, we obtain

$$
\left\|(\log |D|)^{k} g_{\lambda} \star u\right\|_{L^{q}\left(\mathbb{R}^{2 N}\right)} \leq\left\|(\log |D|)^{k} g_{\lambda}\right\|_{L^{r}\left(\mathbb{R}^{2 N}\right)}\|u\|_{L^{p}\left(\mathbb{R}^{2 N}\right)},
$$

with $\frac{1}{r}=\frac{1}{q}-\frac{1}{p}+1 \cdot$ We are then reduced to prove that

$$
\left\|(\log |D|)^{k} g_{\lambda}\right\|_{L^{r}\left(\mathbb{R}^{2 N}\right)} \lesssim \lambda^{k} \mathrm{e}^{2 N \lambda b\left(\frac{1}{p}-\frac{1}{q}\right)} .
$$

Obviously

$$
\mathcal{F}\left((\log |D|)^{k} g_{\lambda}\right)(\xi)=\lambda^{k}\left(\lambda^{-1} \log |\xi|\right)^{k} \phi\left(\lambda^{-1} \log |\xi|\right),
$$

thus in view of the relation $\frac{1}{r}=\frac{1}{q}-\frac{1}{p}+1$, our purpose is to establish that the function

$$
g_{k, \lambda}(x):=(2 \pi)^{-2 N} \int_{\mathbb{R}^{2 N}} \mathrm{e}^{i x \cdot \xi} \phi_{k}\left(\lambda^{-1} \log |\xi|\right) d \xi
$$

with $\phi_{k}(\rho)=\rho^{k} \phi(\rho)$, satisfies $\left\|g_{k, \lambda}\right\|_{L^{r}\left(\mathbb{R}^{2 N}\right)} \lesssim \mathrm{e}^{2 N \lambda b\left(1-\frac{1}{r}\right)}$.

To this end, we shall follow the strategy adopted in the proof of Lemma 1.12, Firstly since the function $\phi_{k}$ belongs to $\mathcal{D}(] 1, b[)$, we infer that

$$
\left|g_{k, \lambda}(x)\right| \lesssim \int_{1}^{\mathrm{e}^{\lambda b}}\left|\phi_{k}\left(\lambda^{-1} \log \rho\right)\right| \rho^{2 N-1} d \rho
$$

which gives rise to

$$
\left\|g_{k, \lambda}\right\|_{L^{\infty}\left(\mathbb{R}^{2 N}\right)} \lesssim \mathrm{e}^{2 N \lambda b} .
$$

This ends the proof of the result in the case when $r=\infty$.

Recall that the case when $r=1$ corresponds to the case studied in Lemma 1.12. Thus to achieve our goal, it suffices to consider the case when $1<r<\infty$. Taking advantage of (2.9), we deduce that, for any positive real number $\delta$, the following estimate holds

$$
\int_{|x| \leq \delta}\left|g_{k, \lambda}(x)\right|^{r} d x \lesssim\left(\delta \mathrm{e}^{\lambda b r}\right)^{2 N}
$$

Moreover according to (2.3) and (2.4), we have

$$
\left.|| x\right|^{2 N} g_{k, \lambda}(x) \mid \lesssim \lambda^{-1} \text { and }\left.|| x\right|^{2 N+1} g_{k, \lambda}(x) \mid \lesssim \lambda^{-2} .
$$


We deduce that

$$
\begin{aligned}
\int_{|x| \geq 1}\left|g_{k, \lambda}(x)\right|^{r} d x & =\left.\left.\int_{|x| \geq 1}|| x\right|^{2 N+1} g_{k, \lambda}(x)|| g_{k, \lambda}(x)\right|^{r-1} \frac{d x}{|x|^{2 N+1}} \\
& \lesssim \lambda^{-2} \mathrm{e}^{2 N \lambda b(r-1)}
\end{aligned}
$$

and for any $0<\delta \leq 1$

$$
\begin{aligned}
\int_{\delta \leq|x| \leq 1}\left|g_{k, \lambda}(x)\right|^{r} d x & =\left.\left.\int_{\delta \leq|x| \leq 1}|| x\right|^{2 N} g_{k, \lambda}(x)|| g_{k, \lambda}(x)\right|^{r-1} \frac{d x}{|x|^{2 N}} \\
& \lesssim \lambda^{-1} \mathrm{e}^{2 N \lambda b(r-1)} \int_{\delta}^{1} \frac{d \rho}{\rho} \lesssim-\lambda^{-1} \mathrm{e}^{2 N \lambda b(r-1)} \log (\delta) .
\end{aligned}
$$

Selecting $\delta=\mathrm{e}^{-\lambda b r}$ achieves the proof of the lemma.

\section{Logarithmic Sobolev embeddings}

3.1. Sobolev embedding of logarithmic Besov spaces into the Orlicz spaces. The following result, which is an immediate consequence of Proposition 4.1 in [10, improves the Sobolev embedding (1.3). We sketch its proof here for the reader's convenience.

Proposition 3.1. There is a positive constant $C$ such that

$$
\|w\|_{\mathcal{L}\left(\mathbb{R}^{2 N}\right)} \leq C\left\||D|^{N} w\right\|_{B_{2, \infty}^{\left.0, \log _{(\mathbb{R}}{ }^{2 N}\right)}} .
$$

Remark 3.2. As shown by estimates (1.4) and (1.6), the classical Besov space $B_{2, \infty}^{N}\left(\mathbb{R}^{2 N}\right)$ does not embed in $\mathcal{L}\left(\mathbb{R}^{2 N}\right)$.

Proof. To go to the proof of Proposition 3.1 let us split the function $w$ into two parts as follows:

$$
w=w_{1}+w_{2}
$$

where $w_{1}:=\Theta(D) w$, with $\Theta$ a function of $\mathcal{D}\left(\mathbb{R}^{2 N}\right)$ identically equal to 1 near the unit ball. Since for functions in $L^{2}\left(\mathbb{R}^{2 N}\right) \cap L^{\infty}\left(\mathbb{R}^{2 N}\right), \mathcal{L}\left(\mathbb{R}^{2 N}\right.$ ) behaves like $L^{2}\left(\mathbb{R}^{2 N}\right)$ (see for instance [7] for further details), we infer that

$$
\left\|w_{1}\right\|_{\mathcal{L}\left(\mathbb{R}^{2 N}\right)} \leq C\left\|w_{1}\right\|_{L^{2}\left(\mathbb{R}^{2 N}\right)} .
$$

To handle $w_{2}$, let us for fixed $\lambda>0$ estimate the integral:

$$
\int_{\mathbb{R}^{2 N}}\left(\mathrm{e}^{\left|\frac{w_{2}(x)}{\lambda}\right|^{2}}-1\right) d x
$$

Obviously

$$
\int_{\mathbb{R}^{2 N}}\left(\mathrm{e}^{\left|\frac{w_{2}(x)}{\lambda}\right|^{2}}-1\right) d x=\sum_{p \geq 1} \frac{\left\|w_{2}\right\|_{L^{2 p}}^{2 p}}{\lambda^{2 p} p !} .
$$

Firstly let us investigate $\left\|w_{2}\right\|_{L^{2 p}}^{2 p}$. Knowing that for any $p \geq 1$

$$
\left\|w_{2}\right\|_{L^{2 p}}^{2 p} \leq C^{2 p}\left\|\widehat{w_{2}}\right\|_{L^{\frac{2 p}{2 p-1}}}^{2 p},
$$

we are led to estimate $\left\|\widehat{w_{2}}\right\|_{L^{\frac{2 p}{2 p-1}}}$. For that purpose, let us write

$$
\widehat{w_{2}}(\xi)=\frac{1}{|\xi|^{2 N}} \widetilde{w}(\log |\xi|, \omega) .
$$

Observing that

$$
\left\||D|^{N} w_{2}\right\|_{B_{2, \infty}^{0, \log \left(\mathbb{R}^{2 N}\right)}} \sim \sup _{j \in \mathbf{Z}} \int_{2^{j-1}}^{2^{j+1}} \int_{\mathbb{S}^{2 N-1}}|\widetilde{w}(t, \omega)|^{2} d t d \omega
$$

and

$$
\left\||D|^{N} w_{2}\right\|_{L^{2}\left(\mathbb{R}^{2 N}\right)} \sim \int_{0}^{\infty} \int_{\mathbb{S}^{2 N-1}}|\widetilde{w}(t, \omega)|^{2} d t d \omega,
$$


we deduce from Hölder inequality that for any $p \geq 2$

$$
\left\|\widehat{w_{2}}\right\|_{L^{\frac{2 p}{2 p-1}}}^{\frac{2 p}{2 p-1}} \lesssim\left\||D|^{N} w_{2}\right\|_{B_{2, \infty}^{0,10}\left(\mathbb{R}^{2 N}\right)}^{\frac{2 p}{2 p}} \sum_{j \in \mathbf{Z}}\left(\int_{2^{j-1}}^{2^{j+1}} \mathrm{e}^{-\frac{2 N t}{p-1}} d t\right)^{\frac{p-1}{2 p-1}} .
$$

By straightforward computations, we find that

$$
\left(\int_{2^{j-1}}^{2^{j+1}} \mathrm{e}^{-\frac{2 N t}{p-1}} d t\right)^{\frac{p-1}{2 p-1}}=\left(\frac{p-1}{2 N}\right)^{\frac{p-1}{2 p-1}}\left(\mathrm{e}^{\frac{-2 N 2^{j-1}}{p-1}}-\mathrm{e}^{\frac{-2 N 2^{j+1}}{p-1}}\right)^{\frac{p-1}{2 p-1}} .
$$

Taking advantage of the fact that the ratio $\frac{p-1}{2 p-1}$ is uniformly bounded with respect to $p \geq 2$, we deduce that

$$
\left(\int_{2^{j-1}}^{2^{j+1}} \mathrm{e}^{-\frac{2 N t}{p-1}} d t\right)^{\frac{p-1}{2 p-1}} \lesssim(2 p-1)^{\frac{p-1}{2 p-1}} \mathrm{e}^{\frac{-2 N 2^{j-1}}{2 p-1}}
$$

Choosing $j_{0}$ so that $\frac{1}{2} \leq \frac{2^{j_{0}}}{2 p-1} \leq 1$, we infer that

$$
\begin{aligned}
\sum_{j \geq j_{0}}\left(\int_{2^{j-1}}^{2^{j+1}} \mathrm{e}^{-\frac{2 N t}{p-1}} d t\right)^{\frac{p-1}{2 p-1}} & \lesssim(2 p-1)^{\frac{p-1}{2 p-1}} \sum_{j \geq j_{0}} \mathrm{e}^{-\frac{2 N 2^{j}}{2 p-1}} \\
& \lesssim(2 p-1)^{\frac{p-1}{2 p-1}} \sum_{j \geq j_{0}} 2^{-j}(2 p-1) \\
& \lesssim(2 p-1)^{\frac{p-1}{2 p-1}} 2^{-j_{0}}(2 p-1) \lesssim(2 p-1)^{\frac{p-1}{2 p-1}}
\end{aligned}
$$

Making use again of the fact that the ratio $\frac{p-1}{2 p-1}$ is uniformly bounded with respect to $p \geq 2$, we obtain for $j \leq j_{0}$

$$
\begin{aligned}
\left(\int_{2^{j-1}}^{2^{j+1}} \mathrm{e}^{-\frac{2 N t}{p-1}} d t\right)^{\frac{p-1}{2 p-1}} & =\left(\frac{p-1}{2 N}\right)^{\frac{p-1}{2 p-1}} \mathrm{e}^{-\frac{2 N 2^{j+1}}{2 p-1}}\left(\mathrm{e}^{\frac{3 N 2^{j}}{p-1}}-1\right)^{\frac{p-1}{2 p-1}} \\
& \lesssim(2 p-1)^{\frac{p-1}{2 p-1}}\left(\frac{2^{j}}{2 p-1}\right)^{\frac{p-1}{2 p-1}} \lesssim\left(2^{j}\right)^{\frac{p-1}{2 p-1}},
\end{aligned}
$$

which gives rise to

$$
\sum_{j \leq j_{0}}\left(\int_{2^{j-1}}^{2^{j+1}} \mathrm{e}^{-\frac{2 N t}{p-1}} d t\right)^{\frac{p-1}{2 p-1}} \lesssim \sum_{j \leq j_{0}}\left(2^{\frac{p-1}{2 p-1}}\right)^{j} \lesssim 2^{\left(j_{0}+1\right) \frac{p-1}{2 p-1}} \lesssim(2 p-1)^{\frac{p-1}{2 p-1}} .
$$

We deduce that for any $p \geq 2$

$$
\left\|w_{2}\right\|_{L^{2 p}}^{2 p} \lesssim C^{2 p}\left\||D|^{N} w_{2}\right\|_{B_{2, \infty}^{0, \log _{(}}\left(\mathbb{R}^{2 N}\right)}^{2 p-1)^{p-1}} .
$$

Along the same lines, we obtain

$$
\left\|w_{2}\right\|_{L^{2}}^{2} \lesssim\left\||D|^{N} w_{2}\right\|_{B_{2, \infty}^{0, \log }\left(\mathbb{R}^{2 N}\right)}^{2}
$$

which leads to

$$
\int_{\mathbb{R}^{2 N}}\left(\mathrm{e}^{\left|\frac{w_{2}(x)}{\lambda}\right|^{2}}-1\right) d x \lesssim \sum_{p \geq 1} \frac{C^{2 p}\left\||D|^{N} w_{2}\right\|_{B_{2, \infty}^{0, \log _{(}\left(\mathbb{R}^{2 N}\right)}}^{2 p}(2 p-1)^{p-1}}{\lambda^{2 p} p !} .
$$

In view of Stirling formula, we infer that

$$
\left\|w_{2}\right\|_{\mathcal{L}\left(\mathbb{R}^{2 N}\right)} \leq C\left\||D|^{N} w_{2}\right\|_{B_{2, \infty}^{\left.0, \log _{(\mathbb{R}}{ }^{2 N}\right)}},
$$

which in view of (3.1) achieves the proof of the result. 
3.2. A refined radial estimate. The following result is the counterpart of the well-known radial estimate away from the origin available for any radial function in $H^{1}\left(\mathbb{R}^{2 N}\right)$ :

$$
|u(x)| \leq \frac{C_{2}}{\sqrt{|x|^{2 N-1}}}\|u\|_{L^{2}\left(\mathbb{R}^{2 N}\right)}^{\frac{1}{2}}\|\nabla u\|_{L^{2}\left(\mathbb{R}^{2 N}\right)}^{\frac{1}{2}} .
$$

Proposition 3.3. There is a positive constant $C$ such that for any radial function $w$, we have

$$
\sup _{0<|x| \leq \mathrm{e}^{-1}} \frac{|w(x)|}{\sqrt{-\log |x|}} \leq C\|w\|_{\dot{H}^{N}\left(\mathbb{R}^{2 N}\right)}^{\frac{1}{2}}\left\||D|^{N} w\right\|_{B_{2, \infty}^{0, \log _{(}}\left(\mathbb{R}^{2 N}\right)}^{\frac{1}{2}} .
$$

Proof. To go to the proof of the radial estimate (3.6), let us as in the proof of Proposition 3.1, split the function $w$ into two parts as follows:

$$
w=w_{1}+w_{2}
$$

where $w_{1}=\Theta(D) w$. Obviously

$$
\left\|w_{1}\right\|_{L^{\infty}\left(\mathbb{R}^{2 N}\right)} \lesssim\left\|w_{1}\right\|_{L^{2}\left(\mathbb{R}^{2 N}\right)} .
$$

Now arguing as in the proof of Proposition 3.1, write

$$
\widehat{w_{2}}(\xi)=\frac{1}{|\xi|^{2 N}} \widetilde{w}(\log |\xi|) .
$$

Observing that for any $0<|x| \leq \mathrm{e}^{-1}$, there exists $p \in \mathbf{N}$ such $\mathrm{e}^{-2^{p+1}} \leq|x| \leq \mathrm{e}^{-2^{p}}$, let us decompose $w_{2}(x)$ as follows

$$
w_{2}(x)=W^{(1)}(x)+W^{(2)}(x)
$$

with $W^{(1)}(x):=\frac{1}{(2 \pi)^{2 N}} \int_{1 \leq|\xi| \leq \mathrm{e}^{p+1}} \frac{\mathrm{e}^{i x \cdot \xi}}{|\xi|^{2 N}} \widetilde{w}(\log |\xi|) d \xi$.

To estimate the part $W^{(1)}$, we shall perform the change of variable $t=\log |\xi|$ and make use of Cauchy-Schwarz inequality which give rise to

$$
\begin{aligned}
\left|W^{(1)}(x)\right| & \leq \frac{1}{(2 \pi)^{2 N}} \int_{1 \leq|\xi| \leq \mathrm{e}^{2^{p+1}}} \frac{|\widetilde{w}(\log |\xi|)|}{|\xi|^{2 N}} d \xi \\
& \lesssim \sum_{j=-\infty}^{j=p} \int_{2^{j}}^{2^{j+1}}|\widetilde{w}(t)| d t \\
& \lesssim \sum_{j=-\infty}^{j=p} 2^{\frac{j}{2}}\left(\int_{2^{j}}^{2^{j+1}}|\widetilde{w}(t)|^{2} d t\right)^{\frac{1}{2}} \lesssim 2^{\frac{p}{2}} \sup _{j \in \mathbf{Z}}\left(\int_{2^{j}}^{2^{j+1}}|\widetilde{w}(t)|^{2} d t\right)^{\frac{1}{2}} .
\end{aligned}
$$

This implies in view of (3.2) that

$$
\left|W^{(1)}(x)\right| \leq 2^{\frac{p}{2}}\left\||D|^{N} w_{2}\right\|_{B_{2, \infty}^{0,10 g}\left(\mathbb{R}^{2 N}\right)} .
$$

Consequently, we deduce that for $\mathrm{e}^{-2^{p+1}} \leq|x| \leq \mathrm{e}^{-2^{p}}$

$$
\frac{\left|W^{(1)}(x)\right|}{\sqrt{-\log |x|}} \lesssim \frac{2^{\frac{p}{2}}}{\sqrt{-\log |x|}}\left\||D|^{N} w_{2}\right\|_{B_{2, \infty}^{0, \log }\left(\mathbb{R}^{2 N}\right)} \lesssim\left\||D|^{N} w_{2}\right\|_{B_{2, \infty}^{0, \log \left(\mathbb{R}^{2 N}\right)}},
$$

which achieves the proof of the result for the part $W^{(1)}$ according to the Sobolev embedding

$$
L^{2}\left(\mathbb{R}^{2 N}\right) \hookrightarrow B_{2, \infty}^{0, \log }\left(\mathbb{R}^{2 N}\right) .
$$

To address the part $W^{(2)}$, we shall make advantage of the radial estimate (3.5) which ensures that

$$
\left|W^{(2)}(x)\right| \leq \frac{C}{\sqrt{|x|^{2 N-1}}}\left\|W^{(2)}\right\|_{L^{2}\left(\mathbb{R}^{2 N}\right)}^{\frac{1}{2}}\left\|\nabla W^{(2)}\right\|_{L^{2}\left(\mathbb{R}^{2 N}\right)}^{\frac{1}{2}} .
$$


Obviously, we have

$$
\begin{aligned}
\left\|W^{(2)}\right\|_{L^{2}\left(\mathbb{R}^{2 N}\right)}^{2} & =\frac{1}{(2 \pi)^{2 N}} \int_{|\xi| \geq \mathrm{e}^{2+1}} \frac{|\widetilde{w}(\log |\xi|)|^{2}}{|\xi|^{4 N}} d \xi \\
& \lesssim \sum_{q \geq p+1} \mathrm{e}^{-2 N 2^{q}} \int_{\mathrm{e}^{2^{q}} \leq|\xi| \leq \mathrm{e}^{2^{q+1}}} \frac{|\widetilde{w}(\log |\xi|)|^{2}}{|\xi|^{2 N}} d \xi \\
& \lesssim \sum_{q \geq p+1} \mathrm{e}^{-2 N 2^{q}} \sup _{q \in \mathbf{Z}} \int_{2^{q}}^{2^{q+1}}|\widetilde{w}(t)|^{2} d t \lesssim \mathrm{e}^{-2 N 2^{p+1}}\left\||D|^{N} w_{2}\right\|_{B_{2, \infty}^{0,10 g}\left(\mathbb{R}^{2 N}\right)}^{2}
\end{aligned}
$$

Along the same lines

$$
\begin{aligned}
\left\|\nabla W^{(2)}\right\|_{L^{2}\left(\mathbb{R}^{2 N}\right)}^{2} & =\frac{1}{(2 \pi)^{2 N}} \int_{|\xi| \geq \mathrm{e}^{2^{p+1}}} \frac{|\widetilde{w}(\log |\xi|)|^{2}}{|\xi|^{4 N-2}} d \xi \\
& \lesssim \mathrm{e}^{-2(N-1) 2^{p+1}} \int_{|\xi| \geq \mathrm{e}^{2 p+1}} \frac{|\widetilde{w}(\log |\xi|)|^{2}}{|\xi|^{2 N}} d \xi \\
& \lesssim \mathrm{e}^{-2(N-1) 2^{p+1}}\|\widetilde{w}\|_{L^{2}}^{2} .
\end{aligned}
$$

Taking advantage of (3.3), we infer that

$$
\left\|\nabla W^{(2)}\right\|_{L^{2}\left(\mathbb{R}^{2 N}\right)} \lesssim \mathrm{e}^{-(N-1) 2^{p+1}}\left\||D|^{N} w_{2}\right\|_{L^{2}\left(\mathbb{R}^{2 N}\right)} .
$$

This leads to the following estimate for $\mathrm{e}^{-2^{p+1}} \leq|x| \leq \mathrm{e}^{-2^{p}}$

$$
\begin{aligned}
\left|W^{(2)}(x)\right| & \lesssim \frac{\mathrm{e}^{-\frac{(2 N-1) 2^{p+1}}{2}}}{\sqrt{|x|^{2 N-1}}}\left\||D|^{N} w_{2}\right\|_{L^{2}\left(\mathbb{R}^{2 N}\right)}^{\frac{1}{2}}\left\||D|^{N} w_{2}\right\|_{B_{2, \infty}^{0, \log }\left(\mathbb{R}^{2 N}\right)}^{\frac{1}{2}} \\
& \lesssim\left\||D|^{N} w_{2}\right\|_{L^{2}\left(\mathbb{R}^{2 N}\right)}^{\frac{1}{2}}\left\||D|^{N} w_{2}\right\|_{B_{2, \infty}^{0, \log }\left(\mathbb{R}^{2 N}\right)}^{\frac{1}{2}},
\end{aligned}
$$

which ends the proof of the proposition.

\section{REFERENCES}

[1] S. Adachi and K. Tanaka, Trudinger type inequalities in $\mathbb{R}^{N}$ and their best exponents, Proceeddings of the American Mathematical Society, 128 (2000), pages 2051-2057.

[2] D. R. Adams, A sharp inequality of J. Moser for higher order derivatives, Annals of Mathematics, 128 (1988), pages 385-398.

[3] H. Bahouri, A. Cohen and G. Koch, A general wavelet-based profile decomposition in the critical embedding of function spaces, Confluentes Mathematici, 3 (2011), pages 387-411.

[4] H. Bahouri, P. Gérard et C.-J. Xu, Espaces de Besov et estimations de Strichartz généralisées sur le groupe de Heisenberg, Journal d'Analyse Mathématique, 82 (2000), pages 93-118.

[5] H. Bahouri et I. Gallagher, Paraproduit sur le groupe de Heisenberg et applications, Revista Matematica Iberoamericana, 17 (2001), pages 69-105.

[6] H. Bahouri, J.-Y. Chemin and R. Danchin, Fourier Analysis and Nonlinear Partial Differential Equations, Grundlehren der mathematischen Wissenschaften, Springer, (2011).

[7] H. Bahouri, M. Majdoub and N. Masmoudi, On the lack of compactness in the 2D critical Sobolev embedding, Journal of Functional Analysis, 260 (2011), pages 208-252.

[8] H. Bahouri, M. Majdoub and N. Masmoudi, Lack of compactness in the 2D critical Sobolev embedding, the general case, Journal de Mathématiques Pures et Appliquées, 101 (2014), pages 415-457.

[9] H. Bahouri, On the elements involved in the lack of compactness in critical Sobolev embedding, Concentration Analysis and Applications to PDE, Trends in Mathematics, (2013), pages 1-15.

[10] H. Bahouri and G. Perelman, A Fourier approach to the profile decomposition in Orlicz spaces, Mathematical Research Letters, 21 (2014), pages 1-22.

[11] H. Bahouri, C. Fermanian and I. Gallagher, Refined Inequalities on Graded Lie Groups, Notes aux Comptes-Rendus de l'Académie des Sciences de Paris, Série I 350 (2012), pages 393-397.

[12] I. Ben Ayed and M. K. Zghal, Characterization of the lack of compactness of $H_{r a d}^{2}\left(\mathbb{R}^{4}\right)$ into the Orlicz space, Communications in Contemporary Mathematics, 16 (2014), pages 1-25.

[13] J.-M. Bony, Calcul symbolique et propagation des singularités pour les équations aux dérivées partielles non linéaires, Annales de l'École Normale Supérieure de Paris, 14 (1981), pages 209-246. 
[14] G. Bourdaud, La propriété de Fatou dans les espaces de Besov homogènes, Note aux Comptes Rendus Mathematique de l'Académie des Sciences 349 (2011), pages 837-840.

[15] H. Brézis and J.-M. Coron, Convergence of solutions of H-Systems or how to blow bubbles, Archive for Rational Mechanics and Analysis, 89 (1985), pages 21-86.

[16] G. Furioli, C. Melzi and A. Veneruso, Littlewood-Paley decompositions and Besov spaces on Lie groups of polynomial growth, Mathematische Nachrichten, 279 (2006), pages 1028-1040.

[17] I. Gallagher and Y. Sire, Besov algebras on Lie groups of polynomial growth, Studia Math, 212 (2012), pages 119-139.

[18] P. Gérard, Description du défaut de compacité de l'injection de Sobolev, ESAIM Contrôle Optimal et Calcul des Variations, 3 (1998), pages 213-233.

[19] S. Jaffard, Analysis of the lack of compactness in the critical Sobolev embeddings, Journal of Functional Analysis, 161 (1999), pages 384-396.

[20] S. Klainerman and I. Rodnianski, A geometric approach to the Littlewood-Paley theory, Geometric and Functional Analysis, 16 (2006), pages 126-163.

[21] P.-L. Lions, The concentration-compactness principle in the calculus of variations. The limit case. I., Revista Matematica Iberoamericana 1 (1) (1985), pages 145-201.

[22] P.-L. Lions, The concentration-compactness principle in the calculus of variations. The limit case. II., Revista Matematica Iberoamericana, 1 (2) (1985), pages 45-121.

[23] J. Moser, A sharp form of an inequality of N. Trudinger, Indiana University Mathematics Journal, 20 (1971), pages 1077-1092.

[24] M.-M. Rao and Z.-D. Ren, Applications of Orlicz spaces, Monographs and Textbooks in Pure and Applied Mathematics, 250 (2002), Marcel Dekker Inc.

[25] B. Ruf, A sharp Trudinger-Moser type inequality for unbounded domains in $\mathbb{R}^{2}$, Journal of Functional Analysis, 219 (2005), pages 340-367.

[26] I. Schindler and K. Tintarev, An abstract version of the concentration compactness principle, Revista Mathematica Complutense, 15 (2002), pages 417-436.

[27] B. Ruf and F. Sani, Sharp Adams-type inequalities in $\mathbb{R}^{n}$, Transactions of the American Mathematical Society, 365 (2013), pages 645-670.

[28] H. Triebel, Theory of Function Spaces, Birkhäuser.

(H. Bahouri) Laboratoire D’Analyse et de Mathématiques Appliquées UMR 8050, Université Paris-Est Créteil, 61, avenue du Général de Gaulle, 94010 Créteil Cedex, France

E-mail address: hbahouri@math.cnrs.fr 